\title{
Oncological cases and complications in Urology
}

\author{
Massimiliano Bernabei ${ }^{1}$, Antonia Di Domenico ${ }^{2}$, Gil Falcao ${ }^{3}$, Charalampos Fragkoulis ${ }^{4}$, \\ Andrea Benelli ${ }^{2}$, Martina Beverini ${ }^{2}$, Luís Campos Pinheiro ${ }^{3}$, Cabrita Carneiro ${ }^{3}$, Nicolò Fabbri ${ }^{5}$, \\ Ioannis Glykas ${ }^{4}$, Salvatore Greco ${ }^{6}$, Carlo Introini ${ }^{2}$, Konstantinos Ntoumas ${ }^{4}$, Georgios Papadopoulos ${ }^{4}$, \\ Mariangela Rutigliani ${ }^{7}$, Panagiotis Stamatakos ${ }^{4}$, João Vasco Barreira ${ }^{8}$ \\ ${ }^{1}$ Department of Urology, Azienda USL di Ferrara, Lagosanto, Italy; \\ ${ }^{2}$ Urology Department, E.O. Galliera, Genoa, Italy; \\ ${ }^{3}$ Urologist, Lisbon, Portugal; \\ ${ }^{4}$ Urology Department, General Hospital of Athens "G. Gennimatas", Athens, Greece; \\ ${ }^{5}$ Unit of General Surgery, Azienda Unità Sanitaria Locale di Ferrara, Lagosanto, Italy; \\ ${ }^{6}$ Department of Morphology, Surgery and Experimental Medicine, University of Ferrara, Ferrara, Italy; \\ ${ }^{7}$ Pathology Unit, E.O. Galliera, Genoa, Italy; \\ ${ }^{8}$ Medical Oncologist, Lisbon, Portugal.
}

\section{Summary This collection of cases describes some unusual urological tumors and complica-} tions related to urological tumors and their treatment.

Case 1: A case of left hydronephrosis referred four years after a right radical mastectomy for lobular breast carcinoma was described. Computed tomography scan revealed a left hydronephrosis with dilated ureter up to the proximal third. An exploratory laparoscopy was performed and the definitive histopathology examination showed a recurrence of the carcinoma with a right tubal metastasis and peritoneal carcinosis.

Case 2: A rare case of an extensive penile squamous cell carcinoma in a young man. The patient was treated with radical surgery and modified inguinal lymphadenectomy. No recurrence was noticed so far.

Case 3: A rare case of left sided Inferior Vena Cava (IVC) in a patient diagnosed with renal cell cancer who underwent open left partial nephrectomy.

Case 4: A case of urethrorrhagia, caused by a recent trauma from an urinary catheter placed in a patient submitted to gastric resection due to a neoplastic pathology. Urethrorrhagia only temporarily responded to conservative treatment and ultimately resolved by coagulation with an endoscopic approach.

KEY WORDS: Penile cancer; HPV; Rare tumors; Urethrorrhagia; Endoscopy; Coagulation.

Submitted 26 July 2020; Accepted 28 September 2020

\section{Case 1 \\ HYDRONEPHROSIS AS UNUSUAL PRESENTATION OF METASTATIC LOBULAR BREAST CANCER}

(Di Domenico Antonia, Benelli Andrea, Beverini Martina, Rutigliani Mariangela, Introini Carlo)

\section{INTRODUCTION}

Breast cancer is currently the most common malignancy in women, with an observed incidence rate of 127.5 cases per 100.000 women per year according to the Surveillance, Epidemiology and End Results (SEER) pro- gram. Invasive lobular carcinoma (ILC) accounts for 14\% of all breast cancers; however due to the large incidence ILC affects a large number of women.

Metastatic spread is the single most significant predictor of poor survival. Patients with distant metastases have a five-year survival rate of 27\% (2008-2014). Typical breast cancer metastatization sites are bones, liver, lungs and brain, but many other localizations have been described in literature, including gynecological organs and peritoneum. Fallopian tube metastasis from non-gynecological malignancies are rarely described in literature especially from breast cancer (1). The aim of this report is to describe our experience regarding an unusual case of breast cancer metastasis in a middle age woman focusing on the diagnosis process and clinical management.

\section{Case report}

A 61-year-old woman with a history of lobular breast cancer presented incidental finding of left hydronephrosis at a CT scan of the chest during oncological follow-up. This patient was treated 4 years before with right radical mastectomy and subsequent adjuvant ormonotherapy and radiotherapy. In her history there was a subtotal hysterectomy with finding of uterine carcinoma in situ. The subsequent CT scan of the abdomen showed a bilateral hydroureteronephrosis with an unclear thickening of the distal ureteral wall. A thin circumferential thickening of the rectum was also described. Due to these findings a diagnostic ureteroscopy and a colonscopy were perfomed; a biopsy of the suspicious tissue was performed and in both cases the pathology results were negative. A repeat CT scan of the abdomen after 2 months revealed circumferential thickening of the rectum and the last ileal loop, bilateral hydronephrosis, thickening of wide ligaments with inhomogeneity of the annexial region bilaterally. A large peritoneal "sausage"- shaped mass was described. The patient underwent a PET-CT showing a increased uptake of the intestine areas but turned out to be negative in the pelvis. On physical examination the patient was found to have a palpable mass in the right upper quadrant

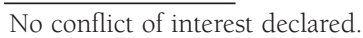




\section{Case 1 - Figure 1.}

A) Metastatic lobular carcinoma of breast growing beneath tubal epithelium. (Hematoxylin-eosin staining magnification 20x). B) Immunohistochemical Estrogen slide showing diffuse and intense positive of the majority of tumor cells (Estrogen, magnification 20x). C) Metastatic lobular carcinoma: immunostaining with GATA 3 shows a characteristic canalicular pattern (GATA3, magnification 20x). D) The Ki-67 proliferation index is 20\% (KI-67 Mib1, magnification 20x).

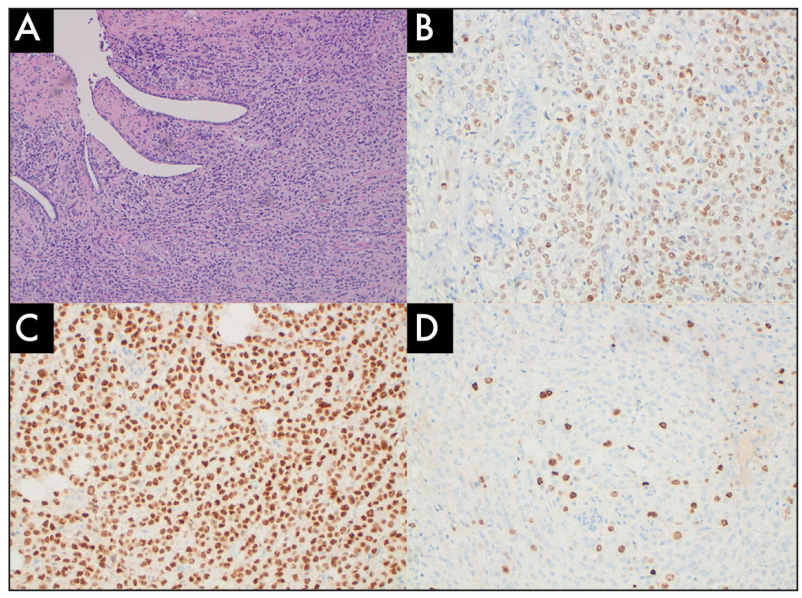

of the abdomen. An exploratory laparoscopy was than performed with evidence of intense pelvic tissue thickening, in particular a mass was evidenced in right pelvis that as first hypothesis was compatible with hydrosalpinx. Peritoneal and cecal nodules were biopsied. Macroscopic examination of the resected specimen showed fallopian tube congestion with lumen in part ectasic in part reduced and thickened and edematous wall. Microscopic section showed small and relatively uniform tumor cells growing in single line and solid. The mitotic index was 3 mitoses/10 high power fields (HPF). By immunohistochemistry, the tumor cells were positive for cytokeratin 7 (clone SP52, prediluited, Ventana), GATA 3 (clone L50-823, prediluited, Cell Marque), Estrogen (clone SP1, prediluited, Ventana), Progesterone (clone 1E2, prediluited, Ventana). They were negative for HER 2 (clone 4B5, 1/100, Ventana), E-cadherin (EP700Y, prediluited, Cell Marque) cytokeratin 20 (clone SP33 prediluited, Ventana). Ki-67 (clone 30-9, prediluited, Ventana) immunostaining showed nuclear labeling in 20\% of the cells. We diagnosed metastases of invasive lobular breast cancer to the fallopian tube (Figure 1). Actually the patient is alive and undergoing chemotherapy with partial radiologic regression of the peritoneal mass. She still needs bilateral double J ureteral stents in order to protect the renal function during chemotherapy.

\section{Conclusions}

Breast cancer is the most common site-specific cancer in women and is the leading cause of death from cancer for patients aged 20-59 years. The interval between diagnosis of primary and metastatic disease is described in literature between 5 and 20 years; in our case the presentation was after 4 years from the primary treatment (2). Metastatic patterns of breast carcinoma have been studied, suggesting two important factors impacting the site of tumor spread: the estrogen receptor (ER) status and the pathology of cancer (ductal versus lobular). Lobular car- cinoma is not the most common histological subtype of breast carcinoma, but it is the most frequent related to gastrointestinal, gynecological and peritoneal metastases. In a series of 100 cases, Rabban et al. found that the two main primary tumors metastasizing to the fallopian tube were colon (35\%) and the breast (15\%) (3).

Carcinomatosis can occur in $2.6 \%$ of cases. The literature rarely mention peritoneal metastasis from breast cancer, and peritoneal recurrences are described after a variable time interval between 5 and 10 years from the diagnosis of primary breast cancer. This late onset might not always be related to a late metastasis development rather than to a late metastasis detection, which results very difficult in the absence of any specific symptom. Moreover, modern imaging techniques resulted scarcely accurate in peritoneal carcinomatosis diagnosis (2). To our knowledge, this is the first reported case of tubal metastasis and peritoneal carcinomatosis from a lobular breast cancer diagnosed by an incidental finding of hydronephrosis. This case emphasizes the role of the fallopian tubes as a potential conduit between the gynecological tract and the peritoneal cavity.

\section{REFERENCES}

1. Bigorie V, Morice P, Duvillard P, et al. Ovarian metastases from breast cancer: report of 29 cases. Cancer. 2010; 116:799-804.

2. Winston CB, Hadar O, Teitcher JB, et al. Metastatic lobular carcinoma of the breast: patterns of spread in the chest, abdomen, and pelvis on CT. AJR Am J Roentgenol. 2000; 175:795-800.

3. Rabban JT, Vohra P, Zaloudek CJ. Nongynecologic metastases to fallopian tube mucosa: a potential mimic of tubal high-grade serous carcinoma and benign tubal mucinous metaplasia or nonmucinous hyperplasia. Am J Surg Pathol. 2015; 39:35-51.

\section{Case 2}

\section{SquAMOUS CELl CARCINOMA OF THE PENIS (Gil Falcão, Cabrita Carneiro, Luís Campos Pinheiro, João Vasco Barreira)}

\section{INTRODUCTION}

Penile cancer is uncommon. The incidence of penile cancer increases with age with a peak in the sixth decade, but it does occur in younger men. About one third of cases are attributed to HPV-related carcinogenesis. Squamous cell carcinoma accounts for over 95\% of penile malignancies. Early inguinal lymphadenectomy in clinically node-negative patients is superior for longterm patient survival compared to later lymphadenectomy with regional nodal recurrence. Patient education is an essential part of follow-up. In patients with long-term survival after penile cancer treatment, sexual dysfunction, voiding problems and cosmetic penile appearance may adversely affect the patient's quality of life (QoL). Since penile cancer is rare, patients should be referred to a center with experience and expertise in local treatment, pathological diagnosis, chemotherapy and psychological support for penile cancer patients.

\section{Case report}

The authors present a clinical case of a man in the fifth decade of life with no comorbidities. The presenting fea- 
Case 2 - Figure 1.

A-B: At clinical examination complete dissociation of foreskin with exposure of corpora cavernosa, lesion of bulky dimension about 5-10 cm, no palpable inguinal lymph nodes, purulent exudate.
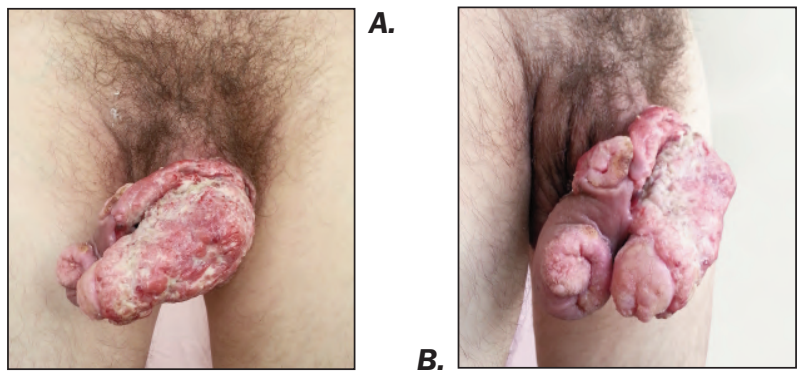

ture was a 4-years onset history of a penile lesion. At the clinical examination we highlight complete dissociation of foreskin with exposure of corpora cavernosa, lesion of bulky dimension about $5-10 \mathrm{~cm}$, no palpable inguinal lymph nodes, purulent exudate (Figure 1).

The histopathological findings of a biopsy of the lesion were compatible with a squamous cell carcinoma (SCC) with overexpression of p16. Staging was performed with thoraco-abdominal-pelvic computed tomography (CT) which revealed a voluminous penile mass, centered on the dorsum of the penis, involving cavernous bodies, spongy body and urethra; the mass contacted the scrotum, with no evident cleavage plan and with no distant sign of disease. At the multidisciplinary evaluation, the patient was proposed for surgical approach.

The histopathological report of the radical penectomy with modified radical lymphadenectomy revealed a squamous cell carcinoma pT3G2NO, R0.

\section{Discussion}

In industrialized countries, penile cancer is uncommon. The incidence of penile cancer increases with age with a peak in the sixth decade, but it does occur in younger men. About one third of cases are attributed to HPVrelated carcinogenesis. A significantly better five-year disease-specific survival has been reported for HPV-related compared with HPV-negative cases. Squamous cell carcinoma accounts for over $95 \%$ of penile malignancies. It is not known how often SCC is preceded by premalignant lesions. Early inguinal lymphadenectomy in clinically node-negative patients is superior for long-term patient survival compared to late lymphadenectomy for regional nodal recurrence. Local recurrence is easily detected by physical examination, by the patient himself or his physician. Patient education is an essential part of follow-up. In patients with long-term survival after penile cancer treatment, sexual dysfunction, voiding problems and cosmetic penile appearance may adversely affect the patient's quality of life (QoL). However, there is very few data on sexual function and QoL after treatment for penile cancer. Since penile cancer is rare, patients should be referred to a center with experience and expertise in local treatment, pathological diagnosis, chemotherapy and psychological support for penile cancer patients. This case is a reminder of how the perception of individual skin findings can lead to the diagnosis of malignant tumors. It also embodies a rare clinical observation, which justifies reporting this case with the purpose to highlight the importance of a multidisciplinary team in the management of cancer patients (1).

\section{REFERENCES}

1. Joura E, Jenkins D, Guimera N. Vulvar, penile, and scrotal human papillomavirus and non-human papillomavirus cancer pathways, In D. Jenkins, F. Xavier Bosch (Eds) Human Papillomavirus providing and using a viral cause for cancer, Academic Press 2020, pages 219-230.

\section{Case 3}

\section{LEFT SIDED INFERIOR VENA CAVA IN A PATIENT DIAGNOSED WITH RENAL CELL CARCINOMA}

(Charalampos Fragkoulis, Ioannis Glykas, Panagiotis Stamatakos, Georgios Papadopoulos, Konstantinos Ntoumas)

\section{INTRODUCTION}

Inferior vena cava (IVC) is a large retroperitoneal vein responsible for the deoxygenated blood transportation from the lower extremities and the abdomen through diaphragm to the right atrium. Anatomically is formed by the confluence of the right and left iliac veins at the level of L5 vertebrae. After its formation it lies along the right anterolateral aspect of the vertebral column and passes through the central tendon of the diaphragm around the T8 vertebral level (1). Many anatomic anomalies of the IVC are described. Most clinically significant are left sided IVC, double IVC, intrahepatic IVC agenesis and total absence of infrarenal IVC (2). Left sided IVC has a suspected prevalence up to $0.5 \%$ and it is caused by the regression of the right supracardinal vein and the persistence of the left supracardinal vein (3).

Injuries of the IVC may occur during operations of the right kidney. Although major vascular injuries involving IVC during laparoscopic or open partial nephrectomy are rare, it is of outmost importance to recognize major anatomic landmarks and to identify anatomic anomalies prior to surgery in order to be able to face any intraoperative complication (4). Therefore, recognizing left sided inferior vena cava variation before surgery through the imaging techniques is very important for the surgeon's preparation of possible vascular damage.

\section{Case report}

We present a case of a 75-year-old male who was referred to our department due to a $5 \mathrm{~cm}$ left renal tumor located in the lower pole. In the CT performed we recognized IVC located in the left of the midline as well as total aplasia of superior vena cava (Figure 1). Vein drainage above the diaphragm was performed through the vein system of azygos and hemiazygos veins. Left renal vein was drained straight to the IVC but as IVC was located to the left side, it did not pass in front of the aorta as usual (Figure 2). A successful open partial nephrectomy was performed by a retroperitoneal approach.

The patient was informed in detail by the treating physician for inclusion in the case presentation and signed an informed consent prior to participation. In the form the 


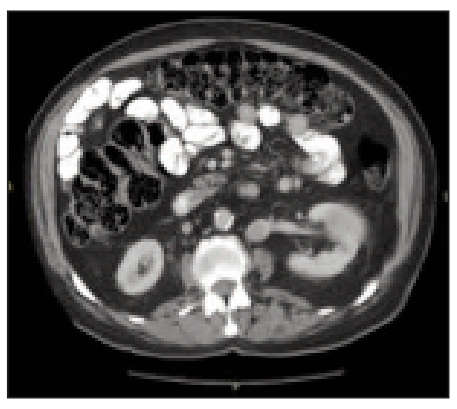

Case 3 - Figure 1. CT showing IVC located in the left of the midline as well as total aplasia of superior vena cava.

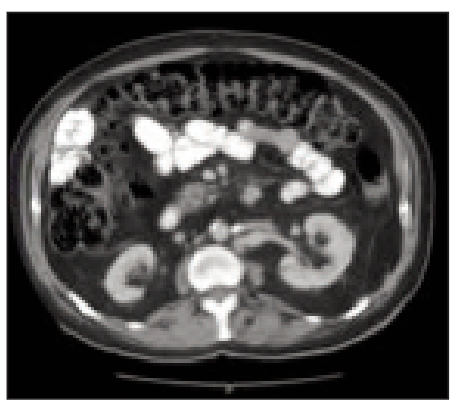

Case 3 - Figure 2.

Left renal vein draining straight to the IVC but as IVC was located to the left side not passing in front of the aorta as usual.

patient has given his consent for his images and other clinical information to be reported in the journal.

The patient understands that his name and initials will not be published.

\section{Conclusions}

Left sided inferior vena cava is an exceedingly rare entity. Recognizing this rare anatomic variation before surgery through the imaging techniques is very important for the surgeon's preparation of possible intraoperative complications.

\section{REFERENCES}

1. Petik B. Inferior vena cava anomalies and variations: imaging and rare clinical findings. Insights Imaging. 2015; 6:631-9.

2. Kaufman JA, Waltman AC, Rivitz SM, Geller SC. Anatomical observations on the renal veins and inferior vena cava at magnetic resonance angiography. Cardiovasc Intervent Radiol. 1995; 18:153-157.

3. Giordano JM, Trout HH. Anomalies of the inferior vena cava.J Vasc Surg. 1986; 3:924-8.

4. McAllister M, Bhayani SB, Ong A, et al. Vena caval transection during retroperitoneoscopic nephrectomy: report of the complication and review of the literature. J Urol. 2004; 172:183-185.

\section{Case 4}

\section{ENDOSCOPIC TREATMENT OF RECURRENT URETHRORRHAGIA}

\section{(Massimiliano Bernabei, Nicolò Fabbri, Salvatore Greco)}

Lesions of the urethra due to urethral catheterization or dislocation of the catheter itself are well known and may sometimes occur with urethrorrhagia and subsequent anemia. The causes may be found in "false paths", stenosis, urethro-penile fistulas, or more rarely, pseudoaneurysms of the bulbar arteries of the penis. The goldstandard treatment consists of percutaneous angioembolization $(1,2)$. We introduce a case of urethrorrhagia, caused by a recent trauma from the urinary catheter, only temporarily responding to conservative treatment and ultimately resolved by the endoscopic approach.

\section{Case report}

A 72-year-old man was admitted to the General Surgery Unit of our hospital in Lagosanto (Ferrara) for gastric resection due to a neoplastic pathology. Before undergoing surgery, he first underwent a cycle of neoadjuvant chemotherapy. In his clinical history, he reported colic diverticulosis, hemorrhoidal syndrome, bilateral inguinal hernioplasty, and mild thrombocytopenia of unknown origin. In the pre-operative period, the patient had reported pollakiuria and dysuria, without requiring urological or medical examinations. The postoperative observation period was complicated with an accidental self-removal of the urinary catheter, accurately positioned before surgery, with a consequent nocturnal episode of urethrorrhagia. The patient did not need the repositioning of the catheter because of spontaneous urination and was discharged after ten days since the operation, with a regular postoperative course and with the urological indication to take tamsulosin $0.4 \mathrm{mg} / \mathrm{day}$ combined with dutasteride $0.5 \mathrm{mg} / \mathrm{day}$ at home. Two days after being discharged, the patient underwent another episode of urethrorrhagia at home, which resolved spontaneously. After three more days (on the $15^{\text {th }}$ postoperative day), the patient presented again to emergency ward for recurrent urethrorrhagia, but this third episode was more persistent and severe. Blood exams documented anemization (hemoglobin levels ranging from 9.4 to $8 \mathrm{~g} / \mathrm{dL}$ in the few hours of stay at the emergency room). It was positioned "per uretram" a latex catheter (20 Ch diameter) and tranexamic acid was administered both locally and orally. Due to only partial resolution of the bleeding, it was admitted to our Urology department. During the hospital stay, the treatment consisted of compression of both penile shaft and perineum with local application of ice; he also needed to be transfused with two units of concentrated red blood cells. After rapid resolution of the urethrorrhagia, a modest urethral bleeding persisted and, in the hypothesis of a lesion of the distal urethra, peri-catheter endo-urethral injection of etilefrine was administered, resulting in an apparent and rapid regression of bleeding.

However, urethrorrhagia presented again two days later, contextually with the evacuation of some clots from the bladder catheter and suprapubic pain symptoms.

We, therefore, decided to consider the patient eligible for urgent operative urethro-cystoscopy, to carry out a bladder washing, waiting for angiography and, if necessary, percutaneous angioembolization. Because of the unavailability of these procedures in our hospital, it was planned to refer the patient to our reference hub hospital in Cona (Ferrara).

The introduction of the cystoscope (Olympus) allowed us to highlight a hyperemic urethral area of about $1 \mathrm{~cm}$, located immediately before the membranous tract of urethra at 7 o'clock position, without evidence of active bleeding. Retrograde endoscopy ruled out further lesions, allowing, once in the bladder, to find and contextually evacuate several intraluminal clots. During the 
Case 4 - Figure 1.

A) Urethroscopy with visualization of the right ventral bulbar urethral lesion, just before the membranous tract.

B) Monopolar coagulation of the margins of the urethral lesion

C) Completion of coagulation D) Final result.
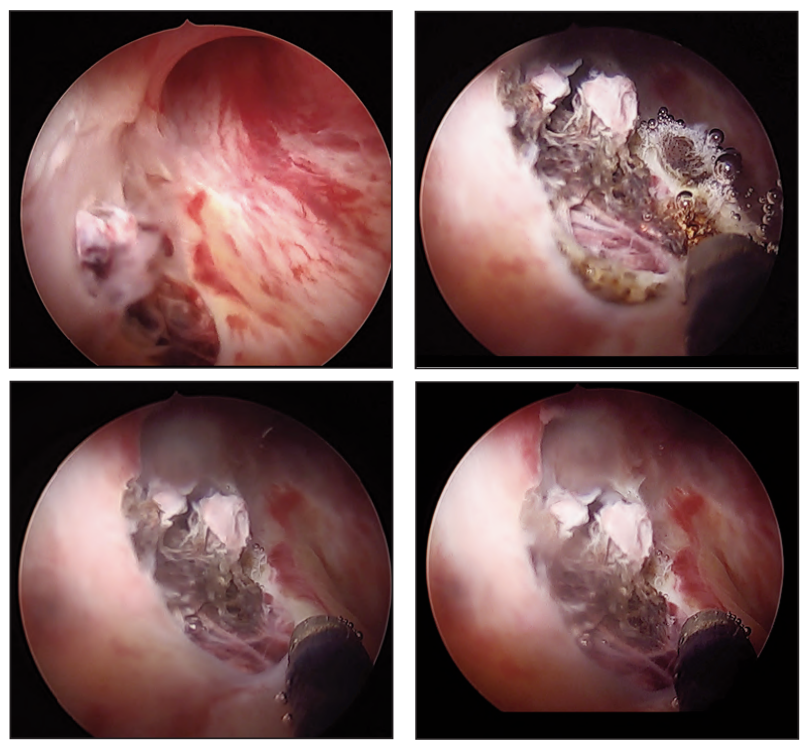

execution of the final antegrade urethroscopy, monopolar diathermocoagulation (DTC) was performed on part of the margin of the a forementioned urethral lesion (Figure 1). After removing the instrument, a two-way "Tiemann" catheter $(20 \mathrm{Ch})$ was then placed. The latter was removed on the third postoperative day, with discharge at home the next day after satisfactory spontaneous normochromic urination. After a further three months of observation, the patient resulted to have no more episodes of urethrorrhagia or abdominal pain symptoms, and the urinary dynamics always remained regular.

\section{Conclusions}

Urethral lesions are well known and frequent in urology, although they result in urethrorrhagia only on rare occasions (3). In cases where this event occurs, conservative treatment (i.e. transurethral catheterization associated with perineal compression) allows resolution in most cases. If this approach is not effective, the more usual alternative is radio-guided angioembolization.

Unfortunately, this procedure may not be readily available in all hospital centers, as it happened for our hospital. To date, only a few reports of endoscopic treatment, as alternative to angioembolization, have been reported in the literature. What we suggest, in the case that radiologicalinterventional treatment (angiography and percutaneous embolization) is not immediately available, is the endoscopic approach. This procedure should aim to endoluminal compression (4), but also to coagulation of any possible active bleeding. Furthermore, in case of significant bleeding, as happened in our case, simple bladder washing may be not effective due to the formation of blood clots that need to be removed with an endoscopic procedure. The "gold standard" treatment should be cystoscopy followed by angiography with embolization; unfortunately, the lack of interventional radiology in our hospital led us to choose an endoscopic attempt to stop bleeding as the first option. In the postoperative period, careful monitoring of the urine color, as well as of the patient's hemodynamic and hematochemical parameters, to exclude any occult bleeding, could be sufficient to avoid further radiological-guided procedures that are not free from possible complications, even functional, as impotence.

\section{REFERENCES}

1. Radhakrishnan S, Marsh R, Sheikh N, et al. Urethral catheter induced pseudoaneurysm of the bulbar artery. Int J Urol. 2005; 12:922-4.

2. Bettez M, Aubé M, Sherbiny ME, et al. A bulbar artery pseudoaneurysm following traumatic urethral catheterization. Can Urol Assoc J. 2017; 11:E47-E49.

3. Campos SJ, Besser PN, Aguirre AP, et al. Urethrorrhagia secondary to traumatic penile pseudoaneurysm. Urol Case Rep. 2016; 7:10-13

4. Schober JP, Iqbal S, Marcantonio A, MacLachlan L. Endoscopic management of a trauma-induced urethral pseudoaneurysm. J Endourol Case Rep. 2019; 5:96.

\section{Correspondence}

Antonia Di Domenico, MD

antonia.didomenico@galliera.it

Andrea Benelli, MD (Corresponding Author)

andrebenne@gmail.com - andrebene85@hotmail.com

Martina Beverini, MD

martina.beverini@live.it

Mariangela Rutigliani, MD

mariangela.rutigliani@galliera.it

Carlo Introini, $M D$

carlo.introini@galliera.it

E.O Galliera, Genoa (Italy)

Gil Falcão, MD (Corresponding Author)

gilfalcao145@gmail.com

Cabrita Carneiro, MD

jpcabritacarneiro@gmail.com

Luís Campos Pinheiro, MD

luiscampospinheiro@gmail.com

João Vasco Barreira, MD

joaovascobarreira@gmail.com

Lisbon, Portugal

\section{Charalampos Fragkoulis, MD}

harisfrag@yahoo.gr

Ioannis Glykas, MD (Corresponding Author)

giannis.glykas@gmail.com

Panagiotis Stamatakos, MD

pvstamatakos@gmail.com

Georgios Papadopoulos, MD

gipapadopoulos@yahoo.gr

Konstantinos Ntoumas, MD

ntoumask@yahoo.com

Urology Department, General Hospital of Athens "G. Gennimatas",

Athens (Greece)

Massimiliano Bernabei, MD

m.bernabei@ausl.fe.it

Nicolò Fabbri, MD (Corresponding Author)

n.fabbri@ausl.fe.it

Salvatore Greco, MD

salvatore.greco@unife.it

Azienda USL di Ferrara, via Valle Oppio, 2, Lagosanto, Italy

University of Ferrara, Ferrara, Italy 